\title{
Trustworthy Institutions in Global Health Research Collaborations
}

\author{
Angeliki Kerasidou
}

\subsection{INTRODUCTION}

Trust is often cited as being fundamental in biomedical research and in research collaborations. However, despite its prominence, its specific meaning and role remain vague. What does trust mean, and is it the same whether directed towards individuals and research institutions? What is it about trust that makes it important in global health research, and how can we effectively promote it? This chapter analyses the meaning of trust and discusses its importance and relevance in the context of global health research collaborations.

In recent decades, biomedical research has moved away from a one-researcher-one-project model to adopt a more collaborative way of working that brings together researchers from different disciplines, institutions and countries. Global health research, a field that has emerged as a distinct area of biomedical research, exemplifies this trend towards collaborative partnerships. Global health, as 'collaborative trans-national research ...,', often relies on collaborations between researchers and institutions from high-income countries (HIC) and low-and-middle income countries (LMIC). LMICs still carry the highest burden of disease globally and have a high prevalence of many illnesses that pose global threats (e.g. infectious diseases). This has motivated a number of national and international funders to support global health research. The redirection of funds towards global health has resulted in increased interest among HIC researchers in working on diseases such as malaria, tuberculosis, HIV-AIDS and conditions such as malnutrition, and also a new impetus in forming partnerships with colleagues from LMICs.

Global health research is seen as a natural field for collaborative work for two reasons. First, by definition, the problems that global health research is trying to answer are complex, multifaceted and transcend borders and boundaries; tackling such problems requires collaborations between disciplines, countries and institutions. Second, most of the issues global health is concerned with affect less affluent parts of the world. Building health research capacity in these countries, and strengthening their public health systems, is seen as the most effective and sustainable way to ensure the successful progress and implementation of global health research, and to meet global health priorities. The importance of trust and the role of institutions in establishing and promoting trust relationships are often noted in discussions regarding global health research

${ }^{1}$ R. Beaglehole and R. Bonita, 'What Is Global Health?', (2010) Global Health Action, 3(1), 5142. 
collaborations. Trust is often presented as a foundational element of research participation, ${ }^{2}$ data sharing ${ }^{3}$ and sharing of samples and other resources. ${ }^{4}$

Here, I give an account of what it means for an institution to be trusted and be trustworthy in the context of global health research. I employ the example of data sharing to illustrate the importance and value of trustworthiness as an institutional moral characteristic. I use the term 'institution' to refer to groups or collectives that actively undertake research, such as universities and research centres. I conclude that trust is important in global health research collaboration because of the power imbalance between partners that often characterises such collaborations. In order to promote trust, institutions need to focus on being trustworthy by developing a behaviour that corresponds to the aims, principles and values they profess to uphold, and by demonstrating that they have incorporated into their functions, rules and regulations the particular needs of their partners and collaborators.

\subsection{WHAT IS A COLLABORATION?}

We use the term 'collaboration' in our everyday language to signify many different types of partnerships. Yet, not all ways of working together are collaborations. The term denotes a particular type of partnership where two or more partners come together to achieve a common aim or goal. ${ }^{5}$ Collaborations are non-hierarchical structures, based on the sharing of decisionmaking and responsibility that rely more on capacity and expertise, rather than on functions or titles. ${ }^{6}$ Consider, for example, a collaboration between a statistics unit and an epidemiology unit working together on a population health project investigating lung cancer. The two groups are committed to the aim of the project and share equal responsibility for its successful completion. They bring different expertise into the project, participate equally in decisions regarding its running and direction and share ownership for its outputs (e.g. authorship on academic publications). Collaborations are characterised by transparency, openness in communication, synergy and honest appreciation of each other's positions. Transparency facilitates a collective awareness of the project, its structure, strengths and weaknesses, and promotes collective ownership. Open communication allows for the free flow of information and exchange of ideas, but also for the expression of concerns. The easier it is for people to talk to each other and share their thoughts and viewpoints, the easier it is for a project to stay on track and reach its goals. Understanding each other's positions and particular circumstances is also important, as it helps with setting expectations at the right level, anticipating problems and foreseeing areas where conflict may arise. Finally, synergy, which describes the drive and desire to achieve the common goal and recognition of the partners' interdependence in fulfilling it, is what drives such partnerships. $^{7}$

\footnotetext{
${ }^{2}$ M. Guillemin et al., 'Do Research Participants Trust Researchers or Their Institutions?', (2018) JEEHRE, 13(3), 285-294.

3 R. Milne et al., "Trust in Genomic Data Sharing among Members of the General Public in the UK, USA, Canada and Australia', (2019) Human Genetics, 138(11-12), 1237-1246.

4 P. Tindana et al., 'Ethical Issues in the Export, Storage, and Reuse of Human Biological Samples in Biomedical Research: Perspectives of Key Stakeholders in Ghana and Kenya', (2014) BMC Medical Ethics, 15(76).

5 E. A. Henneman et al., 'Collaboration: A Concept Analysis', (1995) Journal of Advanced Nursing, 21(1), 103-109; D. D'Amour et al., 'The Conceptual Basis for Interprofessional Collaboration: Core Concepts and Theoretical Frameworks', (2005) Journal of Interprofessional Care, 19(sup 1), 116-131.

${ }^{6}$ Henneman et al., 'Collaboration: A Concept Analysis'.

7 D'Amour et al., 'The Conceptual Basis for Interprofessional Collaboration'.
} 
Other types of partnerships or co-working include cooperation - which brings partners together who do not share the same goal, but who need each other's skills and expertise to reach their individual aims - and hiring or commissioning someone to do a specific job. For example, someone can be brought into a project to complete a very specific task, such as to conduct a systematic literature review, collect samples or develop informed consent forms for a clinical trial. Once the task is completed, the person's involvement in the project is ended. None of these types of partnerships can be described as collaborations as they lack the fundamental characteristics of non-hierarchical and synergistic co-labouring.

\subsection{TRUST AND TRUSTWORTHINESS}

Alongside synergy and horizontal organisation, trust is regularly cited as a fundamental characteristic of collaborations. ${ }^{8}$ D'Amour notes that the term collaboration conveys the idea of sharing ... in a spirit of harmony and trust'. ${ }^{9}$ One empirical study that investigated what underpins successful collaborations in global health research from the perspective of scientists and other research actors, identified trust between partners as one of the major contributing factors. ${ }^{10}$ But why is trust so crucial for collaborations? Trust is yet another term that is often used in our everyday language but not always to describe the same thing. A short analysis would help to better define this term and see how it applies to the context of global health research.

Trust is an attitude towards a person whom we hope, and have good reasons to believe, will behave in a way that confirms our trust. This attitude can take different forms. People can trust others wholeheartedly and perfectly (A trusts B), for example their mother or spouse. But most commonly, trust is perceived as a three-part relationship (A trusts B to $x$ ). There are three main attributes of a trust relationship: vulnerability, assumption of good will from the trustee towards the trustor and voluntariness. ${ }^{11}$ Vulnerability stems from the fact that when trusting, the trustor becomes vulnerable to the trustee as they acknowledge and accept that the trustee can decisively affect the outcome of the entrusted action. ${ }^{12}$ This is what justifies feelings of gratitude or of betrayal when trust is confirmed or broken. ${ }^{13}$ Vulnerability is not, however, a personal characteristic of the trustor. Rather, it is a relational property that emerges from the act of trusting. Consider the following example: a researcher shares some potentially significant pre-publication findings with a colleague who also works in a similar area. She has previously worked with this colleague and trusts him. In her correspondence she stresses the importance of these findings and asks the colleague to keep them confidential. If the colleague confirms her trust, and keeps the findings confidential, she will feel her trust is confirmed; if, however, the colleague ignores her trust and publishes the findings or shares them with others (e.g. at a conference), she will justifiably feel betrayed. The feeling of betrayal is predicated on the fact that she has no assurances, other than her trust, to protect her from the colleague's decision and behaviour,

\footnotetext{
${ }^{8}$ A. W. Pike et al., 'A New Architecture for Quality Assurance: Nurse-Physician Collaboration', (1993) Journal of Nursing Care Quality, 7(3), 1-8; D’Amour et al., 'The Conceptual Basis for Interprofessional Collaboration', 116; M. Parker and P. Kingori, 'Good and Bad Research Collaborations: Researchers' Views on Science and Ethics in Global Health Research', (2016) PLoS ONE 11(10).

9 D'Amour et al., 'The Conceptual Basis for Interprofessional Collaboration'.

10 Parker and Kingori, 'Good and Bad Research Collaborations'.

${ }^{11}$ A. Kerasidou, 'Trust Me, I'm a Researcher!: The Role of Trust in Biomedical Research', (2017) Med Health Care Philos, 20(1), 43-50.

${ }_{12}$ R. Holton, 'Deciding to Trust, Coming to Believe', (1994) Australasian Journal of Philosophy, 72(1), 63-76; S. Wright, 'Trust and Trustworthiness', (2010) Philosophia, 38(3), 615-627.

13 A. Baier, 'Trust and Antitrust', (1986) Ethics, 96(2), 231-260.
} 
and this is what makes her vulnerable towards her trustor. Seeking assurances, by trying to constrain someone's behaviours as a way of limiting one's vulnerability would indicate that the trustor mistrusts, or lacks trust for that person. This is why trusting requires some level of optimism about the trustee or a normative attitude that the trustee ought to do what the trustor wills them to do. ${ }^{14}$

The second characteristic of trust is the belief that the trustee has good will towards the trustor. ${ }^{15}$ It is this belief that counterbalances vulnerability and provides a reasonable justification for trusting someone. To return to the previous example, the researcher reveals the prepublication findings to her colleague because she has good reasons to believe that he has good will towards her and will not intentionally harm or hurt her. If she did not have good reasons to believe this, then choosing to reveal her findings and make herself vulnerable towards him, all things being equal, would be unjustified. Some challenge the importance of good will in trust, by suggesting that trust may be warranted when we believe that those we trust (trustees) will conform to social constraints and norms, or that they will act in the ways we expect out of selfinterest. ${ }^{16}$ Yet, while social constrains and self-interest could increase people's reliability, it is questionable whether such motives can underpin trust. A belief in the good will of the trustee signifies that the trustor has good reason to assume that the trustee cares about her and/or about the things about which she also cares. Although this could be problematic in situations where one does not have insight into the 'psychology of the one-trusted' ${ }^{17}$ one could still justify trust on the belief in the other's good will by adopting a wide notion of good will, which includes commitment to benevolence and conscientious moral attitude. ${ }^{18}$

The third characteristic is that trust is voluntary, insofar as it cannot be forced or demanded. As Baier notes: “Trust me!” is for most of us an invitation which we cannot accept at will either we do already trust the one who says it, in which case it serves at best as reassurance, or it is properly responded to with, "Why should and how can I, until I have cause to?"'19 Trust takes time to establish, and requires an expectation that people will behave not only in the way we assume they will, but rather in the way we assume they should. ${ }^{20}$ A consistent demonstration of good will, as well as capacity to perform the entrusted action, can provide a good reason for trust. And those who want to be trusted can help generate such relationships by fostering and increasing their trustworthiness.

Trustworthiness is a moral characteristic of the trustee and signifies that they have an attitude of good will towards the trustor by being responsive to the trustor's dependency upon them. ${ }^{21}$ The motivation for behaving trustworthily also matters. Trustworthiness signifies something more than just the mere observation of rules and regulations out of self-interest or duty. It is not just a tactic to avoid punishment or penalties, or to fulfil one's sense of duty. Potter describes trustworthiness as a virtue. 'In evaluating someone's trustworthiness', she argues 'we need to know that she can be counted on, as a matter of the sort of person she is, to take care of those

\footnotetext{
${ }^{14}$ V. McGeer, 'Trust, Hope and Empowerment', (2008) Australasian Journal of Philosophy, 86(2), $237-254$.

15 Baier, 'Trust and Antitrust'.

${ }^{16}$ R. Hardin, Trust and Trustworthiness (Russell Sage Foundation, 2002); O. O’Neill, A Question of Trust (Cambridge: Cambridge University Press, 2002).

17 S. Blackburn, Ruling Passion: A Theory of Practical Reasoning (Oxford: Oxford University Press, 1998).

${ }^{18}$ K. Jones, 'Trust as an Affective Attitude', (1996) Ethics, 107(1), 4-25.

19 Baier, 'Trust and Antitrust', 244.

${ }^{\circ}$ M. Urban Walker, Moral Repair: Reconstructing Moral Relations after Wrongdoing (Cambridge University Press, 2006).

${ }^{21}$ K. Jones, 'Trustworthiness', (2012) Ethics, 123(1), 61-85.
} 
things with which we are considering entrusting her' (emphasis added). ${ }^{22}$ However, it is important to note that expectation for one to behave in a certain manner does not compel the trustee to behave in the expected way. The fact that one is being counted on forms an important consideration to be taken into account but does not force one to act in a certain way - otherwise one would be forced to act in a 'trustworthy' way even when the trust placed on one is unjustified or misguided.

So far, I have argued that trust is a relational mode predicated on a reasonable belief in the trustee's skill to perform the entrusted action and also good will towards the trustor. Trust cannot be forced or demanded, and by trusting, one makes oneself vulnerable toward the person they choose to trust. Trustworthiness is a moral characteristic that indicates that someone can be counted on. It is not necessary that a trustworthy person is automatically trusted, but trustworthy behaviour can illicit trust. In the context of these definitions we can explore this chapter's main questions: what is the role of trust in global health research collaborations and can institutions be trusted? If trust is commonly perceived as a characteristic of interpersonal relationships and trustworthiness as a personal quality or virtue, is it possible to talk meaningfully about trusting institutions, or to ascribe moral characteristics such as trustworthiness to collectives?

\subsection{TRUST IN GLOBAL HEALTH RESEARCH}

In 2013, the Council on Health Research for Development (COHRED) published a report on fair collaborations in global health. ${ }^{23}$ It noted that relying on HIC collaborators' good will has not been sufficient to ensure fair and just collaborations between partners. What was needed instead, the report recommended, was to build LMIC institutions' capacity in contract negotiations. The implication seems to be here, that instead of just trusting people to behave fairly and justly and thus opening oneself up to having their trust betrayed, one needs to ensure that people will behave this way. This could be achieved by putting in place contracts that direct and set the parameters of right behaviour. One way of understanding this contractual relationship is as relationship of reliance. Relationships of reliance are based on proven capacity and clear systems of accountability. In such relationships, the expectation is that the partners will act based on selfinterest. Collaborators can ensure successful partnerships by aligning their interests and by putting in place rules to secure against defection. What makes relationships of reliance preferable to relationships of trust is that the former do not require an assumption of good will, nor do they require the trustor to become vulnerable to the trustee. ${ }^{24}$

One important condition, however, must apply for relationships of reliance to work. Reliance requires power parity between partners. ${ }^{25}$ This is because in relationships that operate on selfinterest, it is far easier for the stronger partners to shift the balance to their favour. This is particularly relevant for global health research collaborations, which often bring together institutions from HICs and LMICs. Giving LMIC researchers and institutions the tools to defend and promote their own interests is one way of promoting reliable - rather than trusting partnerships and addressing relationships of dependency, and COHRED's efforts are a valuable step towards this. However, there are a number of reasons why building trust relationships and

\footnotetext{
${ }_{22}$ N. Nyquist Potter, How Can I Be Trusted?: A Virtue Theory of Trustworthiness (Rowman \& Littlefield, 2002 ), p. 7.

${ }^{23}$ COHRED, 'Where There Is No Lawyer: Guidance for Fairer Contract Negotiation in Collaborative Research Partnerships', (COHRED, 2013).

${ }^{24}$ This is not to say that in relationships of reliance things cannot go wrong. One can fail to accurately predict the other person's action, which can result in harm or loss.

25 A. Kerasidou, 'The Role of Trust in Global Health Research Collaborations', (2019) Bioethics, 33(4), 495-501.
} 
promoting trustworthiness remains important in this context. First, in situations where power parity between partners is lacking, trust can be an essential foundation on which to build a good and fair collaboration. For example, attitudes of good will - a crucial feature of trust - can counterbalance self-interested motivations. Second, trust could facilitate good collaborative partnerships, by creating a safe environment in which partners can focus on achieving the common goal rather than on protecting their own interests. Finally, it is common sense that everyone, given the option, would prefer to work with partners they trust and not only with those they can reliably predict their behaviour.

If we accept that trust remains relevant in global health research, what we need to consider next is how it could be promoted. One of the reasons that could justify and encourage a trust relationship is trustworthiness. Although trustworthiness cannot always and de facto guarantee trust, moral agents who want to be trusted by their partners and collaborators could do worse than to try to cultivate and demonstrate their trustworthiness. However, while trustworthiness can be attributed to individual persons, collaborations in global health research however, are not just between individuals, but also between institutions. It is important, therefore, to examine whether it is reasonable to talk about 'trustworthy' institutions.

\subsection{TRUSTWORTHY INSTITUTIONS}

Prior to ascertaining whether institutions (e.g. universities, research centres) can be trustworthy, we must establish whether it is reasonable to talk about groups and collectives possessing such moral characteristics as trustworthiness. ${ }^{26}$ In other words, can institutions involved in global health research collaborations be moral agents? There are two main reasons, which, I believe, give support to the view that collectives are entities that could be treated as moral agents: first, such a position chimes with the way we think about the role of collectives in public life and also the way we treat them in practical terms. For example, we expect universities to adhere to ethical principles when conducting research and we hold them responsible when they fail to do so. ${ }^{27}$ In law, collectives are treated as bearers of rights and responsibilities and can be penalised for wrongdoing and for failing to meet their duties and obligations. Second, the view that institutions are moral agents reflects a growing realisation that many issues require the action of collectives in order to be resolved. Actions such as conducting large-scale research aimed at halting pandemics or reversing climate change are unavailable to individuals but possible to groups and institutions. If we accept that these actions reflect duties that ought to be met, then these duties will have to be ascribed to actors that can meet them. ${ }^{28}$

Being trustworthy means that a given individual (or institution) acts not only as they are expected to, but in a way that demonstrates that they have taken into account the fact that someone is counting on them. ${ }^{29}$ Trustworthiness is a characteristic or moral attitude that is revealed through one's actions and also in one's 'values, commitments and loyalties'.$^{30}$ When it comes to institutions, their trustworthy character is revealed in their professed goals and aims, at

26 The types of groups or collectives I have in mind are those who submit to a common goal, can act as one body and present organisational structures and rules, e.g. universities, research bodies and international agencies, and not those based merely on the sharing of a common characteristic (e.g. a disease).

27 J. Couzin-Frankel, 'A Lonely Crusade', (2014) Science, 344(6186), 793-797; C. Elliot, 'Guinea-pigging', The New Yorker (31 December 2007).

${ }^{28}$ For a comprehensive defence of institutions as moral agents see: C. List and P. Pettit, Group Agency: The Possibility, Design, and Status of Corporate Agents (Oxford University Press, 2011).

${ }^{29}$ Jones, 'Trustworthiness"'; Wright, 'Trust and Trustworthiness'.

$3^{\circ}$ Potter, How Can I Be Trusted?, p. 7 . 
their institutional structures, internal rules and regulations as indicators for their moral motivations, ${ }^{31}$ and in their reputation and track record as indicator for their skill and commitment to right action. ${ }^{32}$ Researchers and groups in global health who are looking for collaborations would perceive institutions that declare to care about things they also care as more trustworthy, rather than institutions that do not profess such interests. An institution's track record and proven capacity in their ability to reach these shared goals would add to its trustworthiness. Importantly, being trustworthy is not about following rules but acquiring a disposition of trustworthiness. Behaving in a certain way only for fear of penalty demonstrates a self-interested orientation, rather than concern for others or about what others value. Therefore, institutions would need to demonstrate that their commitment to trustworthy behaviour is principled and corresponds with their aims and purpose, rather than motivated by a desire to avoid sanctions and penalties, including loss of future collaborations. For institutions participating in global health research, this will mean demonstrating that they have incorporated into their structures, rules and regulations central aims of global health such as addressing health inequalities, improving health through rigorous research and promoting research capacity in countries that lack it. Using the example of data sharing and open access may help to illustrate this point.

\subsection{TRUSTWORTHINESS IN DATA SHARING COLLABORATIONS}

Data sharing is often presented as foundational to global health research. ${ }^{33}$ Health and healthrelated data (e.g. genomic, phenotypic or clinical data) are an inexhaustible resource that could be used repeatedly to address multiple research questions, provide answers to a plethora of global health issues and thereby help reduce the global burden of disease. For example, data sharing between countries and institutions is essential in the attempt to understand and respond to epidemics and pandemics, as the cases of the $\mathrm{H}_{5} \mathrm{~N}_{1}$ avian flu in 2007 and the outbreaks of Ebola in 2014 and ZIKA in 2015 have demonstrated. ${ }^{34}$ Recognition that health data offer valuable resources with multiple applications has led to a position where data sharing is seen as both a scientific and moral imperative in biomedical research, ${ }^{35}$ while failure to share has been variously described as being unscientific, contrary to research integrity, wasteful and unjust. ${ }^{36}$ In recent years, a lot of effort has been put into facilitating and promoting the open sharing of data. ${ }^{37}$ Progress in data sharing tools, methods and policies is seen as the innovation with the farthest-reaching impacts among the global medical community. $3^{8}$ This has led to the wide endorsement of data sharing and open access policies by many international research bodies,

${ }^{31}$ P. A. French, 'Types of Collectivities and Blame', (1975) The Personalist, $56(2), 65-85$; R. Bachmann and A. Inkpen, 'Understanding Institutional-Based Trust Building Processes in Inter-Organizational Relationships', (2011) Organizaition Studies, 32(2), 281-301.

$3^{2}$ Hardin, Trust and Trustworthiness.

33 E. Pisani et al., 'Beyond Open Data: Realising the Health Benefits of Sharing Data', (2016) BMJ, 355.

34 K. Littler et al., 'Progress in Promoting Data Sharing in Public Health Emergencies', (2017) Bulletin World Health Organisation, 95(4), 243-243A.

35 H. Bauchner et al., 'Data Sharing: An Ethical and Scientific Imperative', (2016) JAMA, 315(12), $1238-1240$.

${ }^{6}$ M. Munafo et al., 'Open Science Prevents Mindless Science', (2018) BMJ, 363; P. Langat et al., 'Is There a Duty to Share? Ethics of Sharing Research Data in the Context of Public Health Emergencies', (2011) Public Health Ethics, 4 (1), 4-11; P. C. Gotzsche, 'Why We Need Easy Access to All Data from All Clinical Trials and How to Accomplish It', (2011) Trials, 12(1), 249.

37 M. Wilkinson et al., 'The FAIR Guiding Principles for Scientific Data Management and Stewardship', (2016) Scientific Data, 3 .

$3^{8}$ 'Is Data Sharing a Path to Global Health?', (WIRED, 5 February 2018), www.datamakespossible.westerndigital.com/ data-sharing-panacea-global-health. 
funding organisations, academic publishers and policymakers. ${ }^{39}$ It seems that adopting and promoting open access of data and the implementation of a robust open data sharing policy would signal an institution's moral character as being one dedicated to open, transparent and robust science, and to maximising research benefits for all. But would this mean that such an institution is trustworthy?

In the context of global health research, data sharing can be ethically and practically complex. Despite its potential benefits, there are significant ethical and societal barriers to the wide implementation of open data sharing policies and practices. ${ }^{40}$ Leaving aside confidentiality and consent, a significant issue in global health stems from the uneven ability of institutions in different parts of the world to utilise data. ${ }^{41}$ As Serwadda and others note, advancements in technology that make data collection, storage and sharing easier, and the shift in the social and scientific norms to support openness and sharing, is undermining equitable collaborations between HIC and LMIC. ${ }^{42}$ This has led to 'a landscape, often characterised by limited capacity and deep mistrust, for acceptance and implementation of open data policies'. ${ }^{43}$ Furthermore, despite claims that open data sharing could lead to advancements that would be beneficial to all, including to the communities of origin, this is not always the case. Often, the new therapeutics developed are either too expensive for LMICs to purchase, or these countries lack adequate public health structures to make use of any new actionable knowledge. For example, in 2007, Indonesia refused to share its $\mathrm{H}_{5} \mathrm{~N}_{1}$ avian flu data and samples unless their country was guaranteed affordable access to vaccines - and researchers from other LMIC seem to think that this was a fair response to an unfair situation. ${ }^{44}$ Although data sharing could accelerate the production of new and useful knowledge, it can also contribute to the perpetuation of global injustices and undercut the stated goals of global health research.

Adopting an open access policy to data sharing could make an institution reliable, in the sense that its partners would know what to expect and would be able to predict its behaviour and actions with a certain degree of accuracy. Would recognising this institution's reliability in this domain, however, amount to it been perceived as being trustworthy by its partners? ${ }^{45}$ Although adopting certain (moral) rules and acting consistently is an indication of a certain (moral) character, trustworthiness requires more than that; it requires an attitude of good will and

39 European Medicines Agency, 'European Medicines Agency Policy on Publication of Clinical Data for Medicinal Products for Human Use', (European Medicines Agency, 2014); F. Godlee and T. Groves, 'The New BMJ Policy on Sharing Data from Drug and Device Trials', (2012) BMJ, 345(7884), 10; The Wellcome Trust, Policy on Data Management and Sharing (London, England: The Wellcome Trust, 2009); National Institutes of Health, Final NIH Statement on Sharing Research Data (Bethesda: National Institutes of Health, 2003).

$4^{\circ}$ S. Bull and M. Parker, 'Sharing Public Health Research Data: Towards the Development of Ethical Data-Sharing Practice in Low- and Middle-Income Settings', (2015) Journal of Empirical Research on Human Research Ethics, 10(3), $217-224$

${ }^{41}$ I. Jao et al., 'Research Stakeholders' Views on Benefits and Challenges for Public Health Research Data Sharing in Kenya: The Importance of Trust and Social Relations', (2015) PLoS ONE, 10(9).

42 D. Serwadda et al., 'Open Data Sharing and the Global South - Who Benefits?', (2018) Science, 359(6376), 642-643.

43 Ibid., 642 .

44 K. T. Emerson and M. C. Murphy, 'A Company I Can Trust? Organizational Lay Theories Moderate Stereotype Threat for Women', (2015) Personality and Social Psychology Bulletin, 41(2), 295-307.

45 Hawley argues that drawing a distinction between reliability and trustworthiness of institutions is not useful because 'we can require of our institutions that they are reliable in the respects that matter to us' see: K. J. Hawley, 'Trustworthy Groups and Organisations' in P. Faulkner and T. Simpson (eds), The Philosophy of Trust (Oxford University Press, (2017), p. 20. In her case, Hawley has in mind public institutions with whom 'we' as citizens have a special kind of relationship, meaning that these institutions have a duty to be responsive to our needs and particular circumstances. Whether research institutions have the same duty towards researchers in other countries or to the global research community is not immediately clear. An argument will have to be made to demonstrate that research institutions fall within this special category. However, this investigation falls outside the remit of this chapter. 
responsiveness to the other's needs. Hence, a trustworthy institution in global health research would not blankly endorse an open data sharing policy, but would retain a flexible stance, leaving room for adapting its policies with the specificities of its collaborators in mind. Such adaptations might include time-specific embargoes on data release to give partners a fair head start on using their data, restrictions of use to protect the stated research aims of such partners and embedding contextually meaningful capacity building activities into their collaboration. ${ }^{46}$ Although an open data sharing policy could ensure that maximum value and utility is extracted from data, allowing for the negotiation of a managed access policy would signal an institution with good will towards its collaborators and 'a direct responsiveness to the fact that the other is counting on [it]'. ${ }^{47}$

\subsection{CONCLUSION}

Trust is and will remain important in global health research collaborations, at least until the power imbalance between LMIC- and HIC-based researchers and their institutions is addressed. Institutions committed to advancing the aims of global health, including helping build research capacity in LMIC, should aim to promote fair and trusting collaborations. The best way of achieving this is by cultivating and demonstrating their trustworthiness as a way of eliciting justified trust. Being trustworthy requires more than just the observation of rules or the incorporation of moral principles in policies and structures. Although such moral attitudes would likely increase an institution's reliability, trustworthiness also demands attention to the relational aspect of trust. Trustworthiness requires that the institution is concerned with its partners and what its partners value, acknowledges its partners' vulnerability and demonstrates 'a direct responsiveness to the fact that the other is counting on [it]..$^{8}$ The practice of data sharing provides a useful case to examine what being a trustworthy institution might look like in practice. Moving forward, more research will be required to fully examine the relationship between rules, regulations and policies and the moral character of institutions in global health.

\footnotetext{
${ }^{6}$ M. Parker et al., 'Ethical Data Release in Genome-Wide Association Studies in Developing Countries', (2009) PLOS Medicine, 6(11), e1000143.

47 Jones, 'Trustworthiness', 62.

$4^{8}$ Ibid.
} 